\title{
Postoperative Delirium as a predictor of 90 -day hospital readmission in elderly patients undergoing femoral neck fracture reduction and osteosynthesis
}

\author{
Leonor Silva e Sousa ${ }^{1}$, Sofia Fernandes ${ }^{1}$, Zara Nogueira ${ }^{1}$, Alexandra Resende
}

1 - Anesthesiology Department, Centro Hospitalar Lisboa Norte, Hospital Santa Maria.

\section{BACKGROUND:}

Femoral neck fractures (FNF) are an important cause of hospitalization in elderly population. Delirium is a major complication in this subgroup and its occurrence has been associated with poorer outcome.

AIMS:

1. Assess if postoperative delirium is associated with 90-day hospital readmission after FNF reduction and osteosynthesis (RO)

\section{METHODS:}

WHAT? Observational, retrospective study of patients with FNF undergoing RO surgery alone;

WHEN? From January to December of 2016;

WHERE? In a tertiary university hospital.

Delirium: whenever documented at clinical process: acute cognitive dysfunction and/or psychomotor agitation;

90-day hospital readmission was considered for any cause of hospitalization.

Demographic variables analyzed:

$$
\begin{array}{ll}
\checkmark & \text { Age } \\
\checkmark & \text { Sex } \\
\checkmark & \text { Institutionalization } \\
\checkmark & \text { Previous dementia }
\end{array}
$$

$\checkmark$ Treatment with psychoactive drugs

$\checkmark$ Cardiac Heart Failure

$\checkmark$ Pre-op $\mathrm{Hb}$

$\checkmark$ Pre-op Albumin $\checkmark$ Pre-op and pos-op days of hospitalization

\begin{tabular}{|c|c|c|c|c|}
\hline RESULTS: & $\begin{array}{c}\text { Total } \\
(n=134)\end{array}$ & $\begin{array}{c}\varnothing 90 \text {-day hospital } \\
\text { readmission } \\
(n=99)\end{array}$ & $\begin{array}{c}90 \text {-day hospital } \\
\text { readmission } \\
(n=35)\end{array}$ & $p$ value \\
\hline Age (years) & 83.46 (SD 9.7) & 82.7 (SD 10.4) & 86.4 (SD 6.2) & 0.059 \\
\hline Female sex & $92(68.7 \%)$ & 69 (69.7\%) & $23(65.7 \%)$ & 0.682 \\
\hline Institutionalization & $22(16.4 \%)$ & $14(14.1 \%)$ & $8(22.9 \%)$ & 0.243 \\
\hline Dementia & $29(21.6 \%)$ & $19(19.2 \%)$ & $10(28.6 \%)$ & 0.334 \\
\hline Psychoactive Drugs & 64 (47.8\%) & 48 (48.5\%) & $16(45.7 \%)$ & 0.531 \\
\hline CHF & $34(25.4 \%)$ & $21(21.2 \%)$ & $13(37.1 \%)$ & 0.057 \\
\hline Diabetes & $21(15.7 \%)$ & $13(7.1 \%)$ & $8(22.9 \%)$ & 0.166 \\
\hline Pre-op Hb & 11.5 (SD 1.5) & 11.6 (SD 1.5) & 11.5 (SD 1.4) & 0.714 \\
\hline Pre-op Albumin & 3.49 (SD 0.66) & 3.54 (SD 0.72) & 3.39 (SD 0.49) & 0.295 \\
\hline $\begin{array}{l}\text { Pre-op Days of } \\
\text { Hospitalization }\end{array}$ & 2.98 (SD 3.0) & 3.02(SD 3.2) & 2.79 (SD 2.6) & 0.704 \\
\hline \multicolumn{5}{|l|}{ Anesthesia } \\
\hline General/Combined & 55 & 43 & 12 & 0.349 \\
\hline Delirium & $66(49.2 \%)$ & $42(42.4 \%)$ & $24(68.6 \%)$ & 0.009 \\
\hline $\begin{array}{l}\text { Pos-op Days of } \\
\text { Hospitalization }\end{array}$ & 9.86 (SD 9.2) & 9.56 (SD 8.8) & 10.42 (SD 9.8) & 0.640 \\
\hline Pos-op Complications & $56(41.8 \%)$ & $38(38.4 \%)$ & $18(51.3 \%)$ & 0.261 \\
\hline
\end{tabular}

$\checkmark$ Type of anesthesia

$\checkmark$ Pos-op complications

Univariate analysis with chi-square test; multivariate analysis with binary logistic regression; Statistical significance: $p<0.05$

\begin{tabular}{lccc}
\hline & OR & IC 95\% & $p$ value \\
\hline Age & 1.05 & $0.98-1.11$ & 0.143 \\
\hline Female sex & 0.73 & $0.27-1.95$ & 0.527 \\
\hline Institutionalization & 1.31 & $0.46-3.73$ & 0.614 \\
\hline Delirium & 3.39 & $1.26-9.08$ & 0.015 \\
\hline CHF & 2.26 & $0.88-5.81$ & 0.091 \\
\hline Diabetes & 2.71 & $0.83-8.85$ & 0.099 \\
\hline
\end{tabular}

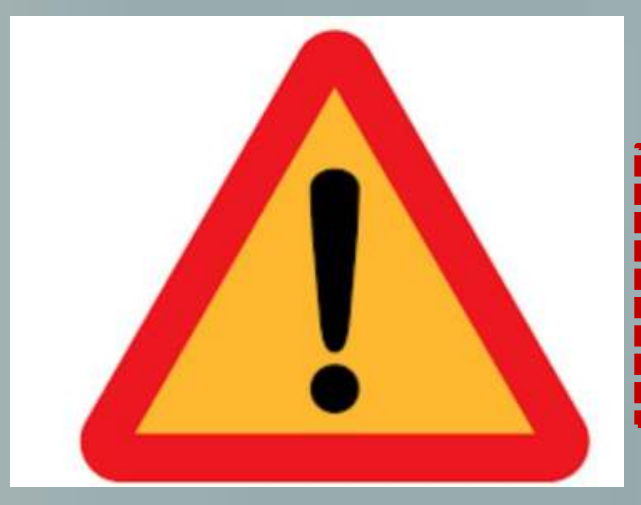

Postoperative delirium was associated with 90-day hospital readmission

\section{CONCLUSION:}

In our cohort of patients undergoing femoral neck fracture reduction and osteosynthesis postoperative delirium is associated with 90-day hospital readmission. 